and allowing for the correlations between bond lengths, the e.s.d's of the mean bond lengths are $0.0028 \AA$ for naphthalene and $0.0021 \AA$ for anthracene. The difference of the means is possibly significant for naphthalene, but, even if the theoretical mean is readjusted, it is still necessary to postulate theoretical errors for this molecule up to about $0 \cdot 020 \AA$ to obtain satisfactory agreement between theory and experiment.

\section{References}

Abrahams, S. C., Robertson, J. M. \& White, J. G. (1949a). Acta Cryst. 2, 233.

Abrahams, S. C., Robertson, J. M. \& White, J. G. (1949b). Acta Cryst. 2, 238.

Ahmed, F. R. \& Cruickshank, D. W. J. (1952). Acta Cryst. 5, 852.

Воотн, A. D. (1945). Nature, Lond. 156, 51.

Booth, A. D. (1946). Proc. Roy. Soc. A, 188, 7.
Coulson, C. A., Daudel, R. \& Robertson, J. M. (1951). Proc. Roy. Soc. A, 207, 306.

Cox, E. G. \& Cruickshank, D. W. J. (1948). Acta Cryst. 1, 92.

Cruickshank, D. W. J. (1949a). Acta Cryst. 2, 65.

Cruickshank, D. W. J. (1949b). Acta Cryst. 2, 154.

Cruickshank, D. W. J. (1952). Acta Cryst. 5, 511.

Cruickshank, D. W. J. \& Rollett, J.S. (1953). Acta Cryst. 6, 705.

Hughes, E. W. (1941). J. Amer. Chem. Soc. 63, 1737.

Krndall, M. G. (1943). The Advanced Theory of Statistics, vol. 1. London: Griffin.

Kendaul, M. G. (1946). The Advanced Theory of Statistics, vol. 2. London: Griffin.

Mathieson, A. McL., Robertson, J.M. \& Sinclatr, V. C. (1950). Acta Cryst. 3, 245.

Sinclarr, V. C., Robertson, J. M. \& Mathinson, A. McL. (1950). Acta Cryst. 3, 251.

WeatherbuRN, C. E. (1947). Mathematical Statistics. Cambridge: University Press.

Acta Cryst. (1953). 6, 705

\title{
Electron-Density Errors at Special Positions
}

\author{
By D. W. J. Crutckshank and J. S. Rollett \\ Chemistry Department, The University, Leeds 2, England
}

(Received 10 February 1953)

\begin{abstract}
Approximate formulae are given for the electron-density and slope errors at special positions in any space group. An example shows that the errors at special positions can be several times those at general positions.
\end{abstract}

\section{Density errors}

The electron density $\varrho(x, y, z)$ is given by the triple Fourier series of structure factors, $F(h k l)$, as

$$
\begin{aligned}
\varrho(x, y, z) & =\frac{1}{V} \sum_{3}|F| \cos \{2 \pi(h x / a+k y / b+l z / c)-\alpha\} \\
& =\frac{1}{V} \sum_{3}|F| \cos (\theta-\alpha), \quad \text { say . }
\end{aligned}
$$

The $F$ 's may be related by symmetry, and in terms of the independent $F$ 's we may write (I) as

$$
\varrho=\frac{1}{V} \underset{\text { indep. }}{\sum}|F| \underset{\text { form }}{\sum} \cos (\theta-\alpha),
$$

where the inner summation is over all planes of the same crystallographic form.

If each independent $|F|$ has a standard deviation $\sigma(F)$, the standard deviation of the error in the electron density, by the law for the combination of errors, is

$$
\sigma(\varrho)=\frac{1}{V}\left\{\sum_{\text {indep. }}^{\Sigma} \sigma^{2}(F)\left[\sum_{\text {form }}^{\Sigma} \cos (\theta-\alpha)\right]^{2}\right\}^{\frac{1}{2}} .
$$

Equation (3) shows that the error varies from point to point in the unit cell. However, if there are a large number of terms in the summation, the error is nearly constant over large regions of the cell, as (3) is the sum of squares of cosine terms. These approximate values of the error depend on whether the position considered is a general one $(x, y, z)$, or a special one, such as $(0,0, z)$ or $(x, \bar{x}, z)$, and on the space group.

For the type of position considered let those planes in each form with the same $|\cos (\theta-\alpha)|$ be said to constitute a sub-form. Let

$$
m=\sum_{\text {sub-form }} \cos (\theta-\alpha) /|\cos (\theta-\alpha)|,
$$

so that if all the planes in a sub-form have the same $\cos (\theta-\alpha), m$ is just the number of planes in that subform. Let $\eta$ be the r.m.s. value of $\cos (\theta-\alpha)$ in a sub-form for positions of the given type; then approximately

$$
\sigma(\varrho)=\frac{1}{V}\left\{\sum_{\text {all sub-forms }}[\eta m \sigma(F)]^{2}\right\}^{\frac{1}{2}},
$$

since, on squaring the summation for each form in (3), 
terms involving products of different $\cos (\theta-\alpha)$ values will have r.m.s. value zero.

For a general $(x, y, z)$ position the sub-forms, except for $F(000)$, will consist only of pairs of terms

$$
\cos (\theta(h k l)-\alpha) \text { and } \cos (\theta(\bar{h} \bar{k} \bar{l})+\alpha),
$$

whatever the space group, and so $m=2$. In these sub-forms the r.m.s. value of $\cos (\theta-\alpha)=1 / \sqrt{ } 2$. For $F(000), m=1$ and $\cos (\theta-\alpha)=1$. Hence

$$
\sigma(\varrho)=\frac{1}{V}\left\{\sigma^{2}(F(000))+\sum_{\text {half lattice }}\left[\frac{1}{\sqrt{ } 2} 2 \sigma\left(F^{\prime}\right)\right]^{2}\right\}^{\frac{1}{2}},
$$

or

$$
\sigma(\varrho)=\frac{1}{V}\left\{\sum_{3} \sigma^{2}(F)\right\}^{\frac{1}{2}}
$$

This is the equation $(11 \cdot 10)$ given by Cruickshank $(1949 a)$, when $\Delta F^{\prime}$ is taken as an estimate of $\sigma(F)$.

\section{Examples}

As an illustration of (5) for a special position, consider the $c$ projection of $P 2_{1} / a$. Here for

$$
\begin{aligned}
& h+k \text { even, } F(h k)=F(h \bar{k}) ; \\
& h+k \text { odd, } \quad F(h k)=-F(h \bar{k}) .
\end{aligned}
$$

For a position of the type $(x, 0), \theta(h k)=\theta(h \bar{k})$, so that the errors for these two planes co-operate when $h+k$ is even $(m=4)$, and cancel when $h+k$ is odd $(m=0)$. Thus the terms in the summation for $\sigma(\varrho)$, corresponding to the four planes of a form $\{h k 0\}$, are

$$
\begin{array}{cc}
h+k \text { even, } & \left(\frac{1}{\sqrt{ } 2} \cdot 4 \sigma(F)\right)^{2} ; \\
h+k \text { odd, } & 0 .
\end{array}
$$

For the pairs of planes of the forms $\{h 00\}, h$ even only, $m=2$ and $\eta=1 / \sqrt{ } 2$. For the forms $\{0 k 0\}$, $k$ even only, $m=2$ and $\cos \theta(0 k)=1=\eta$. Hence

$$
\begin{aligned}
\sigma(\varrho(x, 0))= & \frac{1}{V}\left\{\sigma^{2}(F(000))+\sum_{\{h 00\}}\left[\frac{1}{\sqrt{ } 2} \times 2 \sigma(F(h 00))\right]^{2}\right. \\
& +\sum_{\{0 k 0\}}[1 \times 2 \sigma(F(0 k 0))]^{2} \\
& \left.+\sum_{\substack{\{h k 0\} \\
h+k \text { even }}}\left[\frac{1}{\sqrt{ } 2} \times 4 \sigma(F(h k 0))\right]^{2}\right\}^{\frac{1}{2}} .
\end{aligned}
$$

A more complicated application of (4) was encountered in the analysis of dimethyl triacetylene (Jeffrey $\&$ Rollett, 1952), space group $R \overline{3} m$. The general form $\{h k i l\}$ consists of twelve equivalent planes, all of whose $F$ 's have the same sign. At $(x, y, z)$ the general form splits into six sub-forms of two planes each. At less general positions the sub-forms increase in size, and $\eta=1$ for the sub-forms of some non-general forms. Table 1 gives the contributions to $\sigma(\varrho)$ of the general form for various positions. The last column gives the density errors actually estimated, using $\sigma\left(F^{\prime}\right)$ 's ap-
Table 1. $\sigma(\varrho)$ for dimethyl triacetylene

\section{General form}

$\begin{array}{ccccc}\text { Position } & \begin{array}{c}\text { No. of } \\ \text { sub-forms }\end{array} & \begin{array}{c}\text { Planes in } \\ \text { sub-form }\end{array} & \begin{array}{c}\text { Term in } \\ \sigma(\varrho)\end{array} & \begin{array}{c}\sigma(\varrho) \\ \left(\Theta . \AA^{-3}\right)\end{array} \\ (x, y, z) & 6 & 2 & 6\left(\frac{1}{\sqrt{ } 2} \times 2 \sigma(F)\right)^{2} & 0.018 \\ (x, 0, z) & 6 & 2 & 6\left(\frac{1}{\sqrt{ } 2} \times 2 \sigma(F)\right)^{2} & 0.018 \\ (x, \bar{x}, z) & 3 & 4 & 3\left(\frac{1}{\sqrt{ } 2} \times 4 \sigma(F)\right)^{2} & 0.030 \\ (0,0, z) & 1 & 12 & \left(\frac{1}{\sqrt{ } 2} \times 12 \sigma(F)\right)^{2} & 0.042 \\ (0,0,0) & 1 & 12 & (1 \times 12 \sigma(F))^{2} & 0.053\end{array}$

propriate for the random errors of intensity estimation, and shows that $\sigma(\varrho)$ at a special position can be appreciably greater than at $(x, y, z)$.

\section{Slope errors}

The error in the slope of the electron density is often needed in estimating the error of an atomic coordinate. The slope in the $x$ direction is

$$
\frac{\partial \varrho}{\partial x}=-\frac{1}{V} \frac{2 \pi}{a} \sum_{3} h|F| \sin (\theta-\alpha),
$$

and the standard deviation of the error in the slope is

$$
\sigma\left(\frac{\partial \varrho}{\partial x}\right)=\frac{1}{V} \frac{2 \pi}{a}\left\{\sum_{\text {indep. }}^{\sum} \sigma^{2}(F)\left[\sum_{\text {form }} h \sin (\theta-\alpha)\right]^{2}\right\}^{\frac{1}{2}} .
$$

The approximate value of this is

$$
\sigma\left(\frac{\partial \varrho}{\partial x}\right)=\frac{1}{V} \frac{2 \pi}{a}\left\{\sum_{\text {all sub-forms }}\left[\eta^{\prime} n \sigma(F)\right]^{2}\right\}^{\frac{1}{2}},
$$

where $\eta^{\prime}$ is the r.m.s. value of $\sin (\theta-\alpha)$ in a sub-form averaged over all positions of the given type, and

$$
n=\sum_{\text {sub-form }} h \sin (\theta-\alpha) /|\sin (\theta-\alpha)| .
$$

For a general $(x, y, z)$ position the approximate value in any space group is

$$
\sigma\left(\frac{\partial \varrho}{\partial x}\right)=\frac{1}{V} \frac{2 \pi}{a} \cdot\left\{\sum_{3} h^{2} \sigma^{2}(F)\right\}^{\frac{1}{2}}
$$

(Cruickshank, 1949a, (11.9)). In space groups where no form has terms with the same $|\sin (\theta-\alpha)|$ values, but different $|h|$ values, (9) may be written for any position

$$
\sigma\left(\frac{\partial \varrho}{\partial x}\right)=\frac{1}{V} \frac{2 \pi}{a}\left\{\sum_{\text {all sub-forms }}\left[\eta^{\prime} m^{\prime} h \sigma\left(F^{\prime}\right)\right]^{2}\right\}^{\frac{1}{2}},
$$

where

$$
m^{\prime}=\sum_{\text {sub-form }} \sin (\theta-\alpha) /|\sin (\theta-\alpha)| .
$$

This equation may be used for triclinic, monoclinic and orthorhombic space groups.

An instance of the use of (9) in place of (11) was given by Cruickshank (1949b). 
In the simplest case, when the peaks are well resolved without overlapping in an orthogonal cell of a centrosymmetric structure, the atomic coordinate errors are obtained by dividing the slope error by the peak curvatures. For non-centrosymmetric structures the divisor is a function such as $(3 \cdot 16)$ of Cruickshank (1952). An exact discussion of coordinate errors under general conditions has been given by Cruickshank \& Robertson (1953).

\section{Conditions of validity}

For positions close to but not exactly on special positions the density and slope errors are intermediate between the special and general values, and it may be necessary to use the exact formulae (3) and (8). 'Close' here means within approximately half the distance apart at which two point atoms would be resolved in a similar Fourier analysis containing terms of the same indices.
The approximate formulae also depend on there being a large number of terms in the series, and on there being no plane with $\sigma(F)$ much greater than for any others. The approximate formulae should ordinarily be sufficient if there are more than about 30 different sub-forms.

One of us (J.S.R.) wishes to acknowledge a Further Education and Training Grant from the Ministry of Education, which enabled him to take part in this work.

\section{References}

Cruickshank, D. W. J. (1949a). Acta Cryst. 2, 65. Cruickshank, D. W. J. (1949b). Acta Cryst. 2, 154.

Cruickshank, D. W. J. (1952). Acta Cryst. 5, 511.

Cruickshank, D. W. J. \& Robertson, A.P. (1953). Acta Cryst. 6, 698.

Jeffrey, G. A. \& Rollett, J. S. (1952). Proc. Roy. Soc. A, 213, 86 .

Acta Cryst. (1953). 6, 707

\title{
The Crystal Structure of $\alpha$-Thiopyridone
}

\author{
By Bruce R. Penfold* \\ Crystallographic Laboratory, Cavendish Laboratory, Cambridge, England
}

(Received 4 March 1953)

\begin{abstract}
The crystal structure of $\alpha$-thiopyridone has been determined by means of electron-density projections on the (001) and $(010)$ planes. The $(001)$ projection was solved by direct determination of the signs of Fourier coefficients. Bond lengths of the pyridine ring are interpreted in terms of a resonance structure and hence a value is deduced for the bond order of the $\mathrm{C}-\mathrm{S}$ bond. The $\mathrm{C}-\mathrm{S}$ order/length curve is discussed with reference to the results from this structure analysis and that of thiophthen. $\alpha$-Thiopyridone molecules are linked in pairs across centres of symmetry by what appear to be weak hydrogen bonds between nitrogen and sulphur atoms. There are several points of similarity between this structure and that of $\alpha$-pyridone.
\end{abstract}

\section{Introduction}

In a previous communication (Penfold, 1953), the crystal structure of the tautomeric compound $\alpha$ pyridone was reported, the existence of the pyridone tautomer being established by accurate electrondensity measurements which led to direct location of hydrogen atoms. The molecule of $\alpha$-thiopyridone, which differs from that of $\alpha$-pyridone only in that the oxygen atom has been replaced by a sulphur atom, is also tautomeric in a similar way, the two forms being shown in Fig. 1, I being thiopyridone and II thiolpyridine. It is therefore of some interest to compare its crystal structure with that of $\alpha$-pyridone. The possibility of a reliable determination of the C-S bond length is of some importance because of the addition

* Now at Canterbury University College (University of New Zealand), Christchurch C.1, New Zealand. it would provide to the very meagre body of informa-<smiles>S=c1cccc[nH]1</smiles><smiles>Sc1cccnc1</smiles>

Fig. 1. Tautomeric forms of the molecule. 